\title{
EMPLOYING CARDINAL RANK ORDERING OF CRITERIA IN MULTI-CRITERIA DECISION ANALYSIS
}

\author{
MONA RIABACKE \\ Dept. of Computer and Systems Sciences, Stockholm University, \\ Forum 100, SE-164 40 Kista, Sweden \\ MATS DANIELSON \\ Dept. of Computer and Systems Sciences, Stockholm University \\ Forum 100, SE-164 40 Kista, Sweden \\ ARON LARSSON \\ Dept. of Information Technology and Media, Mid Sweden University \\ SE-851 70 Sundsvall, Sweden \\ LOVE EKENBERG \\ Dept. of Computer and Systems Sciences, Stockholm University \\ Forum 100, SE-164 40 Kista, Sweden
}

\begin{abstract}
The elicitation of preference information in multi-criteria decision analysis (MCDA) processes and the lack of practical means supporting it is a significant problem in real-life applications of MCDA. The issues at hand are problematical in a multitude of ways, but some of these issues may be remedied by accepting weaker input statements from decision-makers than what is most commonly needed, yet being able to utilize these statements for decision evaluation. In this paper, we propose a fast and practically useful weight elicitation method, which builds on the ideas of rank-order methods, but in addition take imprecise cardinal information into account.
\end{abstract}

\section{Introduction}

Multi-Attribute Value Theory, MAVT, and Multi-Attribute Utility Theory, MAUT [1, 2], are the oldest and most widely used MCDA methods in practical applications. The relative importance of each criterion is assessed, as well as, value functions characterizing the satisfaction of the alternatives (according to the decision-maker) under each criterion, and thereafter the overall score of each alternative is calculated. The most common form of value function used is the additive model: $V(a)=\sum_{i=1} w_{i} v_{i}(a)$, where $V(a)$ is the overall value of 
alternative $a, v_{i}(a)$ is the value score reflecting the alternative's performance on criterion $i$, and $w_{i}$ is the weight assigned to criterion $i$.

\section{Weight Elicitation within MCDA}

Many papers have discussed problematic elements of the elicitation process, such as cognitive demand, cf., e.g., [3, 4], difficulties in judging and expressing precise input, cf., e.g., [5, 6], biases, and inconsistencies that arise, cf., e.g., [7, 8]. A systematic mistake in practical applications is the absence of adequate (or the employment of inadequate) weight elicitation techniques [9]. Moreover, providing the required input has been found to be demanding for users of decision analysis tools, and a need for less challenging elicitation methods, which are favoured by users, have been seen as essential for the practical applicability of such tools, cf., e.g., $[10,11]$.

\subsection{Methods for Eliciting Weights in MCDA}

There are many different methods for weight elicitation. Most frequently a precise numerical weight is assigned to each criterion to represent the information extracted from the user. Methods adopting this approach range from simple rating procedures, like the frequently used direct rating (DR) and point allocation (PA) methods, to somewhat more advanced procedures, such as the often used SMART [12], SWING [2], SMARTS [13], and trade-off methods.

Ratio weight procedures maintain ratio scale properties of the decisionmaker's judgments during the elicitation. Accurate determinations of attribute weights by using ratio weight procedures are tricky to acquire in practice as assessed weights are always subject to response error [14]. Consequently, suggestions on how to use imprecise weights have been proposed instead.

The use of surrogate weights, which are derived from ordinal importance information $[3,11,15]$, is one such approach. There are several proposals on how to convert the rankings provided by the decision-maker into numerical weights, e.g., rank sum (RS) weights, rank reciprocal (RR) weights [16], and centroid (ROC) weights [17].

In some decision analysis applications, preferential uncertainties and incomplete information is handled by using intervals, cf., e.g., [18-20], where a range of possible values is represented by an interval. This approach puts less demand on the decision-maker as individual differences in importances and judgments can be represented by value intervals [20]. 


\subsection{Cardinal Rank Ordering of Criteria}

There is a lot of uncertainty involved in elicitation, and the relaxation of precise importance judgments has been found to be advantageous in order to reduce the gap between theory and practice, cf. $[18,21]$. If more precision is needed, the input that needs to be further specified can be identified, which can be a great advantage in real settings.

As mentioned above, to merely provide an ordinal ranking of the criteria is allegedly less demanding on decision-makers and, in a sense, effort-saving. Jia et al. [14] state that due to the relative robustness of linear decision models regarding weight changes, the use of approximate weights often yields satisfactory decision quality, but that the assumption of knowing the ranking with certainty is strong. Instead, they believe that there can be uncertainty regarding both the magnitudes and ordering of weights and that people can be quite confident that some differences in importance are greater than others. Thus, although some weak form of cardinality may exist, cardinal importance relation information is not taken into account in the transformation of rankorders into weights. As an alternative, if ordinal information as well as imprecise cardinality is taken into account, the resulting input is more in line with a reasonable representation of significance.

An elicitation procedure consists of three parts: i) extraction (extracting information through user input); ii) representation (capturing the information in a formal structure); and iii) interpretation (assigning meaning to the captured information). In order to address the issues above, we propose an approach to more pragmatic weight elicitation allowing for ease of use, imprecise weights, and mixing of ordinal and cardinal information as appropriate. The suggested elicitation method, Cardinal Rank Ordering of Criteria (CROC), takes both ordinal information as well as imprecise cardinal relation information of the importance of the attribute ranges into account during extraction, and interprets the criteria input as regions of significance. In its representational and interpretational aspects, CROC extends rank-order methods into handling imprecise cardinal information. If the cardinal information is omitted and only a ranking of criteria is provided, the results coincide with ROC weights [17].

\subsubsection{Extraction}

The method consists of two stages. In the CROC extraction (user interaction) stage, information on the decision-maker's ranking of the criteria in the criteria set is elicited. After the extraction, the information is represented and interpreted in the second stage. In the following, we denote the set of $\mathrm{N}$ criteria $\mathbf{G}=$ 
$\left\{G_{1}, \ldots, G_{N}\right\}$ where each criterion $G_{i} \in \mathbf{G}$ is, during the elicitation, associated with a scale position (value) $x_{i}$ and a criterion weight variable $w_{i}$. The first stage consists of three steps that can be iterated until convergence.

In the first step, only ordinal information is collected. Without loss of generality, we assume that $G_{1}$ is more important than $G_{2}$ which is more important than $G_{3}$ and so forth.

In the second step, the decision-maker is asked to assess the difference in importance between the most and the least important criteria. Denote the highest user score $x_{H}$ and the lowest $x_{L} . x_{H}$ is set to 100 at the outset. The decision-maker is thus asked to express strength in his/her opinion on the difference between the most and least important criteria by setting $x_{L}$ within the interval $(0,100)$.

In the third step, the criteria are initially distributed equally along a cardinal slider according to the ordering obtained in the first step and using the end scale points determined in the second step. The scale position difference between criterion $G_{1}$ and criterion $G_{2}$ is equal to the scale position difference between $G_{2}$ and $G_{3}$, and so on.
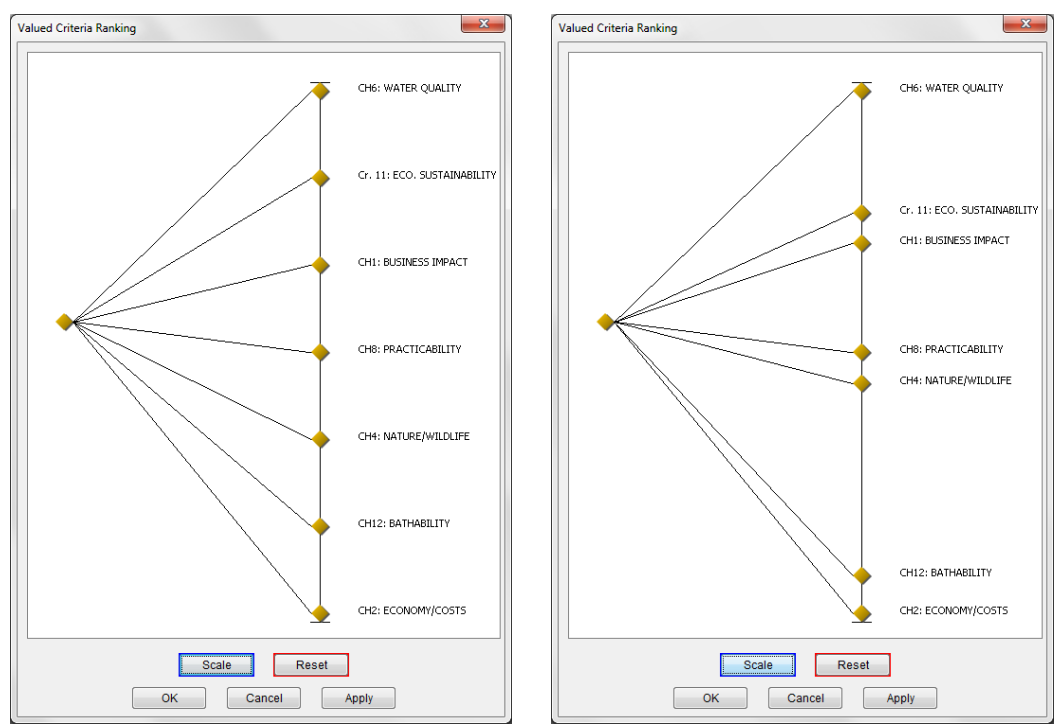

Figure 1. Initial distances (left) and adjusted distances (right) between criteria.

Thereafter, the decision-maker will be able to adjust the distances between the criteria in order to express his or her cardinal importance information between them, e.g., he or she may feel that two criteria are closer together or more apart importance-wise, which is indicated by decreasing or increasing the distance 
between the criteria (see Fig. 1). If the second and third steps are omitted, the procedure coincides with ROC weights.

\subsubsection{Interpretation}

The interpretation stage is concerned with translating the user input from the extraction stage into sets of statements. Underlying the interpretation is the scaling of the user statements from the slider. Assuming normalized criteria weights, i.e. $\Sigma w_{i}=1$ and $0 \leq w_{i} \leq 1$ for all $i \leq \mathrm{N}$, the user information $\left(x_{i} \leq x_{H}\right.$ for all $i \leq \mathrm{N}$ ) from the first stage is mapped onto a normalized Y-scale such that $\Sigma x_{i} \rightarrow 1 ; x_{H} \rightarrow y_{H}=x_{H} / \Sigma x_{i} ; x_{L} \rightarrow y_{L}=x_{L} / \Sigma x_{i}$; and $0 \rightarrow 0$. The middle two are the endpoints of the visible part of the scale; see Fig. 1 for an implementation. Each criterion position $x_{j}$ in between thus maps onto $y_{j}=x_{j} / \Sigma x_{i}$.

In step one of the extraction stage, an ordinal ranking is obtained. Using comparative statements, this is represented as $w_{i} \geq w_{j}$ for $i<j$. In the last step of the extraction, the decision-maker can express cardinality by sliding the different criteria until he/she is satisfied with their relative positions. The end result is then viewed relative to the initial equidistant positions. The interpretation views each scale position as a representative of a region of belief. On the user scale, the criteria are originally spaced $r=\left(x_{H}-x_{L}\right) /(N-1)$ apart. This corresponds to $s=r / \Sigma x_{i}$ on the normalized Y-scale. The user then moves the criteria along the slider to reflect the cardinal differences in importance. For each pair $G_{\mathrm{i}}$ and $G_{\mathrm{j}}$ with $i<j$ of adjacent criteria, let $d_{i j}$ be the movement factor such that $d_{i j}=1$ if the distance was kept the same (i.e. still being $s$ ). Then the new distance is $s \cdot d_{i j}$, implying $d_{i j}<1$ if the criteria were moved closer together and $d_{i j}>1$ if they were moved further apart. More formally, if the new slider positions are $y_{i}^{\prime}$ and $y_{j}^{\prime}$, then $d_{i j}=\left(y_{i}^{\prime}-y_{j}^{\prime}\right) / \mathrm{s}$.

The slider positions of the criteria are then converted into constraints on the weights that represent the importance of the criteria. For each pair $G_{\mathrm{i}}$ and $G_{\mathrm{j}}$ with $i<j$ of adjacent criteria, the initial equidistant positioning reflects the fact that $G_{i}$ is at least as important as $G_{j}$ (represented by $w_{i} \geq w_{j}$ ) but the cardinal positions of the criteria add more information in the form of new constraints. If the decision-maker moved two criteria, this is represented in weight form as $w_{i}$ $w_{j} \geq\left(d_{i j}-1\right) \cdot s$ for each pair of adjacent criteria. The set of inequalities forms an inequality system that defines a polytope. The centroid of the polytope is the weight distribution and can be found by a decision analysis tool such as DecideIT [22]. If the extraction yielded only equidistant criteria, i.e. no cardinal information was supplied, then the CROC centroid coincides with that of ROC 
given that $x_{L}=0$. See Danielson [19] for procedures on expanding centroids to intervals during sensitivity analysis.

\section{Results and Discussion}

The CROC method was employed in a real-life case study (case 1), set up to be comparable to another case study (case 2), where Point Allocation was employed for the elicitation. The decisions faced in cases 1 and 2 were of the same magnitude, influencing tens of thousands of inhabitants in a city council setting. The decisions were "hot issues" that had been debated for a long time (postponed for several years) and were in the hands of the governing politicians. In both cases, the weight elicitation methods (CROC in case 1, and PA in case 2) were employed twice with some time in between elicitation occasions. Case 1 is described in more detail in Danielson et al. [23] and case 2 in Danielson et al. [24].

The settings in the cases 1 and 2 were similar in terms of type of decisionmakers, domain knowledge and decision process, yet these two cases differed a lot. Since the two elicitation procedures employed varied regarding all elicitation components (extraction, representation, and interpretation), it is useful to compare these aspects. The CROC method's extraction part is graphical and its design encourages imprecision, which makes it virtually impossible in practice (other than by chance) to provide the exactly same visual view of criteria preferences on two different occasions. In case 1, statements from both elicitation occasions are represented in the constraint set generated by the interpretation (described in 2.2.2 Interpretation) when changes are minor (which could be attributed to unintended change). In case 2, employing PA, the number of changes were exceedingly more than what would be expected when relating them to the changes in case 1 . This can partly be attributed to the fact that minor (unintended) changes in statements on importance cannot be handled realistically in the interpretation of the method. The vagueness even in the ordinal weight information provided by the decision-makers suggests that there is a need for methods that can handle such input. The outcome of the comparison between the cases indicates that the CROC interpretation is more realistic, and the method more robust and persistent to noisy input. 


\section{References}

1. R. Keeney and H. Raiffa, Decisions with Multiple Objectives: Preferences and Value Trade-offs, (John Wiley, NY, 1976).

2. D. von Winterfeldt and W. Edwards, Decision Analysis and Behavioural Research, (Cambridge University Press, NY, 1986).

3. F. Barron and B. Barrett, Acta Psych. 93, 23-36 (1996).

4. V. Belton and T. Stewart, Multiple Criteria Decision Analysis: An Integrated Approach, (Kluwer Academic Publishers, UK, 2002).

5. Z. Shapira, Risk Taking: A Managerial Perspective, (Russel Sage Foundation, NY, 1995).

6. J. Corner and P Corner, J. Oper. Res. Soc. 46, 304-314 (1995).

7. A. Tversky and D. Kahneman, J. Bus. 59, 252-278 (1986).

8. Lichtenstein, S. and Slovic P. (Eds.), The Construction of Preference, (Cambridge University Press, NY, 2006).

9. J. Pictet and D. Bollinger, Eu. J. Oper. Res. 185, 1300-1307 (2008).

10. P. Bottomley and J. Doyle, Omega 29, 553-560 (2001).

11. K. Katsikopoulos and B. Fasolo, IEEE Trans. Syst. Man Cyber. Part A: Syst. Hum. 36, 960-967 (2006).

12. W. Edwards, IEEE Trans. Syst. Man Cyber. 7, 326-340 (1977).

13. W. Edwards and F. Barron, Org. Beh. Hum. Dec. Proc. 60, 306-325 (1994).

14. J. Jia, G.W. Fischer and J Dyer, J. Beh. Dec. Mak. 11, 85-105 (1998).

15. F. Barron and B. Barrett, Management Science 42, 1515-1523 (1996).

16. W. Stillwell, D. Seaver and W. Edwards, Org. Beh. Hum. Perf. 28, 62-77 (1981).

17. F. Barron, Acta Psych. 80, 91-103 (1992).

18. K.S. Park, IEEE Trans. Syst. Man Cyber. Part A: Syst. Hum. 34, 601-614 (2004).

19. M. Danielson, Int. J. Inf. Tech. Dec. Mak. 3, 513-534 (2004).

20. A. Jiménez, S. Rios-Insua and A. Mateos, A., Comp. Oper. Res. 33, 1081 1101 (2006).

21. A. Larsson, J. Johansson, L. Ekenberg, and M. Danielson, Int. J. Unc. Fuzz. Know.-Based Syst. 13, 495-509 (2005).

22. M. Danielson, L. Ekenberg, J. Johansson and A. Larsson, Proc. of ISIPTA'03, Bernard, J-M., Seidenfeld, T., and Zaffalon, M. (eds.), 204-217, (Carleton Scientific, 2003).

23. M. Danielson, L. Ekenberg, A. Larsson and M. Riabacke, E-Democracy: A Group Decision and Negotiation Perspective, (Rios Insua, D. and French, S., eds., Springer, 2010).

24. M. Danielson, L. Ekenberg, A. Ekengren, T. Hökby and J. Lidén, Journal of Multi-Criteria Decision Analysis 15, 15-30 (2008). 
